Institute for Computational Mathematics

Hong Kong Baptist University

ICM Research Report

09-10 


\title{
Kernel Density Estimation Based Multiphase Fuzzy Region Competition Method for Texture Image Segmentation
}

\author{
Fang $\mathrm{Li}^{\mathrm{a}}$, Michael K. Ng*,b \\ ${ }^{a}$ Department of Mathematics, East China Normal University, Shanghai, China \\ ${ }^{b}$ Centre for Mathematical Imaging and Vision and Department of Mathematics, Hong \\ Kong Baptist University, Kowloon Tong, Hong Kong.
}

\begin{abstract}
In this paper, we propose a multi-phase fuzzy region competition model for texture image segmentation. In the functional, each region is represented by a fuzzy membership function and a probability density function that is estimated by a nonparametric kernel density estimation. The overall algorithm is very efficient as both the fuzzy membership function and the probability density function can be implemented easily. We apply the proposed method to synthetic and natural texture images, and synthetic aperture radar images. Our experimental results have shown that the proposed method is competitive with the other state-of-the-art segmentation methods.
\end{abstract}

Key words: Texture, Multiphase region competition, Kernel density estimation, Fuzzy membership function, Total variation.

\section{Introduction}

Image segmentation is a fundamental task in image processing and computer vision. It is aimed to partition an image into a finite number of subregions with homogeneous intensity (color, texture) properties which will hopefully correspond to objects or object parts. Approaches based on the calculus of variation and partial differential equations (PDEs) are powerful in image segmentation. One important reason of their success is that

\footnotetext{
* corresponding author

Email address: mng@math.hkbu.edu.hk. (Michael K. Ng)
} 
these models are flexible in integrating the geometric information such as shape, length and area. The best known and most influential approaches are Mumford-Shah model [21], geodesic active contour [5], geodesic active region [24], Chan-Vese model [7], region competition [31].

In this paper, we focus on the segmentation of texture images. Piecewise smooth/constant models such as Mumford-Shah model [21] and Chan-Vese model [7] fail in this case. Recently, some variational methods have been proposed to tackle the segmentation of complex textures based on feature extraction techniques $[9,27,25,13]$. In $[9,27]$, a set of Gabor filters with different scales, orientations and frequencies are applied to the image to create the features to represent texture in the image. Chan et al in [9] extended the Chan-Vese model to these vector features for texture image segmentation. Because there are many features to be used in the model, the corresponding minimization method can be slow. Savig et al [27] used the Beltrami framework on the texture features to define a new texture indicator function, and then integrated this function in a combined model of the geodesic active contour [5] and the vectorial Chan-Vese model [7] to segment textural regions. Rousson et al [25] extracted the texture features by applying an anisotropic diffusion process to the structure tensor. In their segmentation framework, a Gaussian approximation is used for all the features channels, and a nonparametric approximation is used for the first gray image channel. The choice of Gaussian approximation restricts the applicability to limited set of images that satisfy the underlying assumption.

Another kind of variational methods for texture image segmentation is based on region competition. Zhu et al [31] proposed a region competition method unifying snake, region growing and Bayesian statistics. It is a parametric model since they assume that each region follows a Gaussian distribution. Kim et al in [17] proposed a nonparametric statistical method for image segmentation using mutual information and curve evolution. However, the above mentioned variational approaches have some practical shortcomings. The above energy functionals are not convex in the optimization space (usually the characteristic functions of sets, which is nonconvex collection) and they have local minima. Typically, the gradient decent method is used in the implementation of these models, and are therefore prone to getting stuck in these local minima. Hence these methods are sensitive to initialization. Meanwhile, the implementation of the above models are based on curve evolution and level set approach [22]. The drawback in the level set implementation consists of initializing the active contour in a distance function and 
re-initializing periodically during the evolution, which is time-consuming.

Based on the observation in the Rudin-Osher-Fatemi [26] model for binary image denoising and Chan-Vese segmentation model, the drawback of leading to local minima comes from the non-convexity of characteristic functions. Recently, Chan et al [10] proposed to use a "segmentation" variable valued in $[0,1]$ to substitute a characteristic function and obtain a new constrained convex functional such that the global minimizer can be achieved in the segmentation process. To make the algorithm more efficient, Bresson et al [2] proposed to add another new variable to approximate "segmentation" variable such that the Chambolle's fast dual projection method [6] can be employed. The advantage of this algorithm is that it is fast and easy to implement. There are several works following this idea [19, 20, 3, 4, 13]. Mory et al $[19,20]$ derived the fuzzy region competition method, parametric and nonparametric statistics error functions in the region terms are considered and studied respectively. Ni et al [4] used histogram and Wasserstein distance in the region term. Houhou et al [13] used shape operator borrowed from different geometry to extract only one texture feature and then used the popular Kullback-Leiber distance in the region term. We remark that the above techniques are only used in two-phase image segmentation.

In [28], Shen developed a multiphase stochastic variational soft segmentation Mumford-Shah model employed a double well potential regularization term in the model. As a set of partial differential equations arising from the model have to be solved, the resulting method is computationally expensive. This method may not be handled a more complicated texture image segmentation problem.

In this paper, we propose a multi-phase fuzzy region competition model for texture image segmentation. In the functional, each region is represented by a fuzzy membership function and a probability density function. The fuzzy membership function is used to represent the region such that the energy functional is convex with respect to membership functions. The probability density function is estimated in each region by using kernel density method. The estimation method is different from $[17,20]$. The overall algorithm is very efficient as both the fuzzy membership function and the probability density function can be implemented easily. We apply the proposed method to synthetic and natural texture images, and synthetic aperture radar images. Our experimental results have shown that the proposed method is competitive with the other state-of-the-art segmentation methods.

The outline of the paper is as follows. We review some related work 
for texture image segmentation in Section 2. In Section 3, we propose our method and the numerical algorithm. Experimental results on various images are showed in Section 4. Finally, we conclude our paper in Section 5.

\section{Related Works}

The general $N$-phase segmentation problem can be formulated as follows: Given an image $I: \Omega \rightarrow \mathbb{R}$ where the image domain $\Omega$ is a bounded, smooth and open subset of $\mathbb{R}^{2}$, the aim is to partition $\Omega$ into $N$ regions $\left\{\Omega_{i}\right\}_{i=1}^{N}$ such that $\Omega_{i} \cap \Omega_{j}=\emptyset, j \neq i$ and $\bigcup_{i=1}^{N} \Omega_{i}=\Omega$ by certain suitable measure. We review some texture image segmentation methods which are closely related to our method.

\subsection{Region Competition}

Zhu and Yuille [31] proposed to minimize the following energy

$$
F\left(\Gamma,\left\{\alpha_{i}\right\}\right)=\sum_{i=1}^{N}\left\{\frac{\mu}{2} \int_{\partial \Omega_{i}} d s-\int_{\Omega_{i}} \log P_{i}\left(I \mid \alpha_{i}\right) d x\right\}
$$

The first term within the braces is the length of the boundary curve $\partial \Omega_{i}$ for region $\Omega_{i} . \quad \Gamma=\bigcup_{i=1}^{N} \partial \Omega_{i}$ is the segmentation boundaries of the entire image. The second term is the sum of the cost for coding the intensity $I$ into region $\Omega_{i}$ by the conditional probability distributions $-\log P_{i}\left(I \mid \alpha_{i}\right)$ where $\alpha_{i}$ is the parameter in the probability density function $P_{i}$. Usually a Gaussian probability density function is considered:

$$
P_{i}\left(I \mid \alpha_{i}\right)=\frac{1}{\sqrt{2 \pi} \sigma_{i}} \exp \left(-\frac{\left(I-\mu_{i}\right)^{2}}{2 \sigma_{i}^{2}}\right),
$$

where $\alpha_{i}=\left(\mu_{i}, \sigma_{i}\right)$ are scalar parameters. Curve evolution technique is used in the implementation to solve the optimization problem in (1).

\subsection{Two Phase Fuzzy Region Competition}

In order to solve a general two-phase region competition problem as minimizing

$$
F\left(\Gamma, \alpha_{1}, \alpha_{2}\right)=\int_{\partial \Omega_{1}} d s+\lambda \int_{\Omega_{1}} r_{1}^{\alpha_{1}} d x+\lambda \int_{\Omega_{2}} r_{2}^{\alpha_{2}} d x
$$


where the image region $\Omega$ is partitioned into $\Omega_{1}$ and $\Omega_{2}, \alpha_{i}$ is the region parameter of the region $\Omega_{i}$, and $r_{i}^{\alpha_{i}}$ is error function, Mory et al [19] proposed to use fuzzy membership function $u \in B V_{[0,1]}(\Omega)$ to represent the region and minimize the two-phase fuzzy region competition energy instead

$$
F\left(u, \alpha_{1}, \alpha_{2}\right)=\int_{\Omega}|\nabla u| d x+\lambda \int_{\Omega} u r_{1}^{\alpha_{1}} d x+\lambda \int_{\Omega}(1-u) r_{2}^{\alpha_{2}} d x .
$$

The fast dual projection method proposed by Chambolle [6] is introduced to solve the problem. The error functions in Chan-Vese model [7] $\left(r_{i}^{\alpha_{i}}=\left(I-c_{i}\right)^{2}\right)$ and the local version are studied in the paper.

In the later work [20], Mory et al considered nonparametric probability density function as an error function and minimize

$$
\begin{aligned}
F\left(u, p_{1}, p_{2}\right)= & \int_{\Omega}|\nabla u| d x+\lambda \int_{\Omega} u \int_{a \in \mathscr{A}}\left(p_{1}(a)-K(I(x)-a)\right)^{2} d a d x \\
& +\lambda \int_{\Omega}(1-u) \int_{a \in \mathscr{A}}\left(p_{2}(a)-K(I(x)-a)\right)^{2} d a d x
\end{aligned}
$$

where $K$ is a symmetric Gaussian kernel

$$
K(z)=\frac{1}{\sqrt{2 \pi} \sigma} \exp \left(-\frac{z^{2}}{2 \sigma^{2}}\right)
$$

and $\mathscr{A} \subset \mathbb{R}$ is the values domain of the image. However, the computation is expensive since it involves the integration in domain $\mathscr{A}$.

\subsection{Nonparametric Method using Mutual Information}

For two-phase image segmentation, Kim et al [17] proposed to minimize the following energy

$$
F(\Gamma)=\int_{\partial \Omega_{1}} d s-\lambda \int_{\Omega_{1}} \log \left(P_{1}\left(I(x), \Omega_{1}\right)\right) d x-\lambda \int_{\Omega_{2}} \log \left(P_{2}\left(I(x), \Omega_{2}\right)\right) d x
$$

where $P_{i}\left(I(x), \Omega_{i}\right) d x=\frac{1}{\left|\Omega_{i}\right|} \int_{\Omega_{i}} K(I(x)-I(\hat{x})) d \hat{x}$ and $K$ is a Gaussian kernel as defined in (4). The last two terms in the right hand side come from the mutual information. Active contour method is used in the formulation. The derived curve evolution equation is quite complicated, especially for multiphase image segmentation. 


\section{The Proposed Method}

\subsection{The Proposed Energy}

We propose to use nonparametric probability density function in the region competition model which energy is

$$
E(\Gamma)=\sum_{i=1}^{N} \frac{\mu}{2} \int_{\partial \Omega_{i}} d s-\sum_{i=1}^{N} \int_{\Omega_{i}} \log P_{i}\left(I, \Omega_{i}\right) d x .
$$

Here $P_{i}\left(I, \Omega_{i}\right)$ is the nonparametric probability density function in region $\Omega_{i}$ which is determined by the pixel values in the region of the image. With a suitable change of the parameter, (5) becomes

$$
E(\chi)=\sum_{i=1}^{N} \int_{\Omega}\left|\nabla \chi_{i}\right| d x-\sum_{i=1}^{N} \lambda \int_{\Omega} \chi_{i} \log P_{i}\left(I, \chi_{i}\right) d x
$$

where $\chi=\left(\chi_{1}, \ldots, \chi_{N}\right)$ is the characteristic function of the region $\Omega_{i}$. Following the idea of [19], we replace the characteristic functions $\chi_{i}$ by fuzzy membership functions $u_{i} \in B V_{[0,1]}(\Omega)$. Note that $B V_{[0,1]}(\Omega)$ is the set of bounded variation functions valued in the interval $[0,1]$. Hence our new fuzzy region competition image segmentation model is to minimize the following energy

$$
E(U, P)=\sum_{i=1}^{N} \int_{\Omega}\left|\nabla u_{i}\right| d x-\sum_{i=1}^{N} \lambda \int_{\Omega} u_{i} \log P_{i}\left(I, u_{i}\right) d x
$$

where the membership functions $\left\{u_{i}\right\}$ satisfies two constraints

$$
\text { (i) } u_{i} \in B V_{[0,1]}(\Omega) \text { and (ii) } \sum_{i=1}^{N} u_{i}=1
$$

and $U=\left(u_{1}, \ldots, u_{N}\right), P=\left(P_{1}, \ldots, P_{N}\right)$. Note that the term $\int_{\Omega}\left|\nabla u_{i}\right| d x$ is a total variation regularization term which is widely used in variational image processing.

For the purpose of efficiency, we follow the idea in $[2,19,20,14]$ and take use of Chambolle's fast dual projection algorithm [6]. For that end we add auxiliary variables $V=\left(v_{1}, \ldots, v_{N}\right)$ and approximate $E(U, P)$ by

$E_{r}(U, V, P)=\sum_{i=1}^{N}\left(\int_{\Omega}\left|\nabla v_{i}\right| d x+\frac{1}{2 \theta} \int_{\Omega}\left(v_{i}-u_{i}\right)^{2} d x-\lambda \int_{\Omega} u_{i} \log P_{i}\left(I, u_{i}\right) d x\right)$

where $\theta$ is chosen small enough such that $u_{i}$ and $v_{i}$ are almost identical with respect to the $L^{2}$ norm. 


\subsection{Energy Minimization}

To find the minimizer of energy $E_{r}$ under the constraint (i) and (ii), we use an alternate minimization method.

\subsubsection{Solving $U$}

First we relax constraint (i) by letting

$$
u_{N}=1-\sum_{i=1}^{N-1} u_{i}
$$

in $E_{r}(U, V, P)$. Then for fixed $P$ and $V$, we solve $U$ by minimizing

$$
E_{1}(\bar{U})=\frac{1}{2 \theta} \sum_{i=1}^{N-1} \int_{\Omega}\left(v_{i}-u_{i}\right)^{2} d x+\lambda \sum_{i=1}^{N-1} \int_{\Omega} u_{i} \log \left(\frac{P_{N}}{P_{i}}\right) d x
$$

subject to

$$
0 \leq u_{i}(x) \leq 1 \text {, for } i=1: N-1 \text {. }
$$

where $\bar{U}=\left(u_{1}, \ldots, u_{N-1}\right)$. Since the objective function is strictly convex and the feasible region is convex, there exists a unique global minimizer $\bar{U}^{*}=\left(u_{1}^{*}, \ldots, u_{N-1}^{*}\right)$ of (10) and the following KKT conditions [? ] are both necessary and sufficient: Suppose $\tilde{U}^{*}$ is the global minimizer of (10), then

(a) $u_{i}^{*}(x) \geq 0,1-u_{i}^{*}(x) \geq 0$

(b) There exist Lagrange multipliers $\beta_{i}^{*}(x)$ and $\gamma_{i}^{*}(x)$ for each point $x \in \Omega$ such that

$$
\frac{\partial E_{1}(\bar{U})}{\partial u_{i}^{*}(x)}=\lambda \log \left(\frac{P_{N}(x)}{P_{i}(x)}\right)+\frac{1}{\theta}\left(u_{i}^{*}(x)-v_{i}(x)\right)=\beta_{i}^{*}(x)-\gamma_{i}^{*}(x)
$$

(c) $\beta_{i}^{*}(x) u_{i}^{*}(x)=0, \gamma_{i}^{*}(x)\left(1-u_{i}^{*}(x)\right)=0$

(d) $\beta_{i}^{*}(x) \geq 0, \gamma_{i}^{*}(x) \geq 0$

for $i=1: N-1$.

First we assume $\tilde{U}=\left(\tilde{u}_{1}, \ldots, \tilde{u}_{N-1}\right)$ satisfies

$$
\lambda \log \left(\frac{P_{N}(x)}{P_{i}(x)}\right)+\frac{1}{\theta}\left(\tilde{u}_{i}(x)-v_{i}(x)\right)=0 .
$$

The solution is

$$
\tilde{u}_{i}=v_{i}-\lambda \theta \log \left(\frac{P_{N}}{P_{i}}\right) .
$$


Then we construct $\hat{u}_{i}$ by projecting $\tilde{u}_{i}$ on $[0,1]$, that is

$$
\hat{u}_{i}:=\min \left\{\max \left\{\tilde{u}_{i}, 0\right\}, 1\right\} .
$$

Let $\eta_{i}(x)=\lambda \log \left(\frac{P_{N}(x)}{P_{i}(x)}\right)+\frac{1}{\theta}\left(\hat{u}_{i}(x)-v_{i}(x)\right)$. For each $x \in \Omega$, we choose $\hat{\beta}_{i}(x)$ and $\hat{\gamma}_{i}(x)$ as follows:

if $\hat{u}_{i}(x) \in(0,1)$, then $\hat{\beta}_{i}(x):=0$ and $\hat{\gamma}_{i}(x):=0$;

if $\hat{u}_{i}(x)=0$, then $\hat{\beta}_{i}(x):=\eta_{i}(x) \geq 0$ and $\hat{\gamma}_{i}(x):=0$;

if $\hat{u}_{i}(x)=1$, then $\hat{\beta}_{i}(x):=0$ and $\hat{\gamma}_{i}(x):=-\eta_{i}(x) \geq 0$.

It is easy to verify that $\left\{\hat{u}_{i}, \hat{\beta}_{i}, \hat{\gamma}_{i}\right\}$ satisfies KKT conditions (a)-(d). Therefore $\hat{U}$ is a minimizer of energy (10) and by uniqueness $\bar{U}^{*}=\hat{U}$.

Then we conclude that the closed form solution of $u_{i}, i=1: N-1$ is given by

$$
u_{i}=\max \left\{\min \left\{v_{i}-\lambda \theta \log \left(\frac{P_{N}}{P_{i}}\right), 1\right\}, 0\right\} .
$$

and $u_{N}$ is given by $(9)$.

\subsubsection{Estimation of $P$}

Fix $U$ and $V$, we estimate the probability density function $P_{i}\left(I, u_{i}\right)$ for class $i$ using the following nonparametric kernel density estimation method. If $x_{1}, x_{2}, \ldots, x_{n} \sim f$ is an independent and identically-distributed sample of a random variable, then the kernel density approximation of its probability density function is

$$
\hat{f}_{h}(x)=\frac{1}{n h} \sum_{i=1}^{n} K\left(\frac{x-x_{i}}{h}\right)
$$

where $K$ is some kernel and $h$ is the bandwidth (smoothing parameter) [23]. In this paper $K$ is taken to be a standard Gaussian function with mean zero and variance 1

$$
K(z)=\frac{1}{\sqrt{2 \pi}} \exp \left(-\frac{z^{2}}{2}\right)
$$

We use the optimal bandwidth [1] for the Gaussian kernel density estimation which is

$$
h=\left(\frac{4}{3 n}\right)^{\frac{1}{5}} \text {. }
$$

Note that different from the proposed method, in [17], the bandwidth is fixed for all regions. The quantity $P_{i}, i=1: N$ is then estimated by formula (12) 
in which the samples are the intensities $I(x)$ of the pixels $x \in \Omega$ where the membership function $u_{i}(x) \geq 0.5$. When the class number $N \geq 3$, we use a non-uniform formula to estimate $P_{N}$ in order to overcome the errors of chosen samples in the $N$ th class. We estimate $P_{N}$ in the term $\log \left(\frac{P_{N}}{P_{i}}\right)$ by formula (12) with samples $I(x)$ satisfying $u_{i}(x)<0.5$.

\subsubsection{Solving $V$}

By fixing $P$ and $U$, the variables $V$ can be solved by minimizing

$$
\int_{\Omega}\left|\nabla v_{i}\right| d x+\frac{1}{2 \theta} \int_{\Omega}\left(v_{i}-u_{i}\right)^{2} d x .
$$

This problem can be efficiently solved by fast duality projection algorithm. The solution is given by

$$
v_{i}=u_{i}-\theta \operatorname{div} p_{i},
$$

where the vector $p_{i}$ can be solved by fixed point method: Initializing $p_{i}^{0}=0$ and iterating

$$
p_{i}^{n+1}=\frac{p_{i}^{n}+\tau \nabla\left(\operatorname{div} p_{i}^{n}-u_{i} / \theta\right)}{1+\tau\left|\nabla\left(\operatorname{div} p_{i}^{n}-u_{i} / \theta\right)\right|}
$$

with $\tau \leq 1 / 8$ to ensure convergence. See [6] for more details.

\subsection{The Algorithm}

The algorithm of minimizing $E$ can be summarized in the following four steps:

- Step 1) Initialize the membership functions $u_{i}, i=1: N$ such that the constraints (A) and (B) are both satisfied.

- Step 2) Estimate $P_{i}$ using (12) with different samples;

- Step 3) Update $v_{i}$ by formula (15) for $i=1: N-1$;

- Step 4) Update $u_{i}$ by formula (11) for $i=1: N-1$ and (9) for $i=N$; Repeat Steps 2)-4) until termination. The termination criterion is as follows:

$$
\left\|U_{\text {new }}-U_{\text {old }}\right\| \leq \epsilon
$$

where $\|\cdot\|$ denotes the Euclidean distance and $\epsilon$ is a small positive number.

Remark that the auxiliary variable $v_{N}$ is not used in the iteration. 


\section{Experimental Results}

We test our algorithm on various images include synthetic texture images, natural images and synthetic aperture radar (SAR) images which appear in the recent image segmentation literatures. We display the segmentation results by paint the contour $u_{i}=0.5(i=1: N-1)$ with different colors on the original image. The membership functions are also displayed for illustration. Our results are compared with other state-of-the-art segmentation methods. Test 1: Fig. 1 shows six synthetic texture images with the two-phase segmentation results by the proposed algorithm. The results are satisfactory.

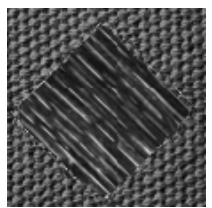

(a)

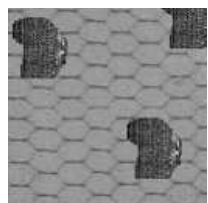

(g)

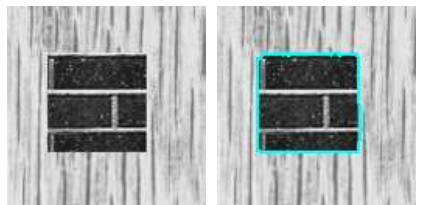

(m)

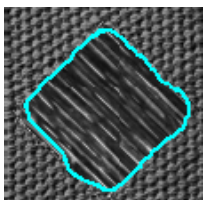

(b)

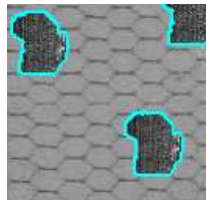

(h)

(n)

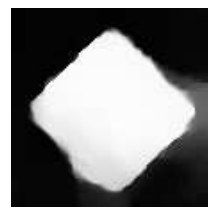

(c)

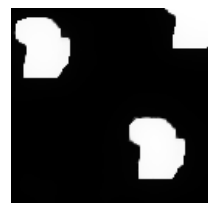

(i)

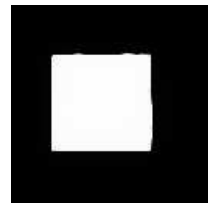

(o)

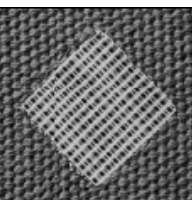

(d)

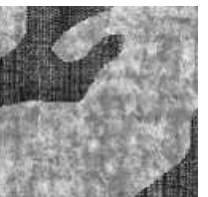

(j)

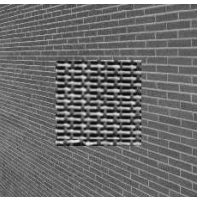

(p)

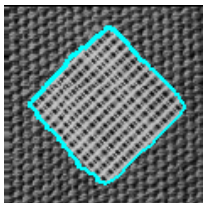

(e)

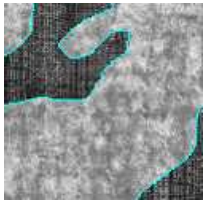

(k)

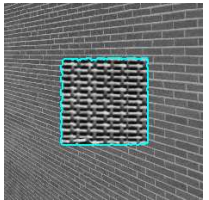

(q)

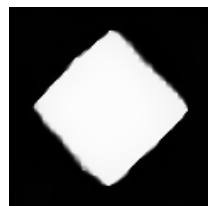

(f)

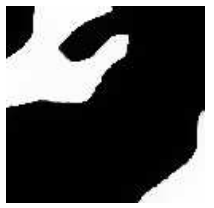

(1)

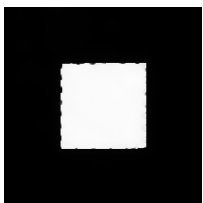

(r)

Figure 1: Two-phase segmentation. The First and Fourth columns: synthetic images; the Second and Fifth columns: segmentation results; the Third and Sixth columns: membership functions $u_{1}$.

Test 2: We test synthetic images generated by several sets of distributions in Fig. 2 as used in [17]. In Fig. 2(a) the two distributions for the foreground and the background are Gaussian with different means and the same variance. In Fig. 2(d) the two distributions for the foreground and the background are Gaussian with the same mean and different variances. For these two 
cases, the method of Yezzi et al. [30] would require the selection of the appropriate statistic (i.e., the means and variances for the first and second cases, respectively) a priori, whereas our method solves the segmentation problem without a prior information. We see from Fig. 2 that the result is as well as that given in [17]. Fig. 2(g) shows a challenging case. The underlying distributions of the foreground and the background are a unimodal Gaussian density and a bimodal density with two Gaussian components as illustrated in Fig. 7(c)-(d) in [17]. The two distributions have the same mean and same variance, so it is hard even for a human observer to separate the foreground from the background. However, our method still gives good segmentation results.

Test 3: In Fig. 3 and Fig. 4, we test our method with 3-phase image segmentation and 4-phase image segmentation respectively. The segmentation results are still satisfactory.

Test 4: In Fig. 5, three natural texture images are tested. Compared with the results of the state-of-the-art works showed in Fig. 6, our results are also satisfactory.

In Fig. 7, we illustrate the evolution of membership function with different initializations for the zebra image in Fig. 5(d). In Fig. 7(a) and Fig. $7(\mathrm{e})$, we initialize $u_{1}=1$ in the white circle, and otherwise $u_{1}=0$. In Fig. 7(i), we set the initial membership function $u_{1}=I / 255$. In Fig. $7(\mathrm{~m})$, we choose the initial membership function randomly valued in $[0,1]$. Fig. 7 shows that our method is not sensitive to initialization which is a drawback of active contour based method. However, the running time of the proposed method depends on the initialization. Good initialization such as Fig. 7(a) converges faster than other initializations. We remark that by experience, the best initialization in terms of speed is obtained by choosing characteristic function of a region inside the object to be segmented.

Test 5: In Fig. 8 and Fig. 9, we make comparison with our method and some other methods for SAR image segmentation. SAR image segmentation is usually said to be a complex problem in the pattern recognition area, due to the presence of speckle derived from the coherency of the image formation process. Fig. 8(a) is a SAR image of trees and grass which has been tested in [15]. They point out that the accurate segmentation of such imagery is quite challenging and cannot be accomplished using standard edge detection algorithms. As illustrated in Fig. 8(b), our method can generate satisfactory segmentation results. Compared with Fig. 8(d) which is the result of method in [15], our method gives a more smooth membership function as shown in 
Fig. 8(c). Fig. 8(e) and Fig. 8(i) are two SAR images tested in [16]. The segmentation results by the multiscale probability neural network method in [16] are shown in Fig. 8(h) and Fig. 8(l). Our method gives more satisfactory results in Fig. 8(g) since the small branches of river are better segmented while in Fig. 8(h) many branches are broken. Our result in Fig. 8(k) has a smooth boundary and is more accurate than Fig. 8(l). Fig. $8(\mathrm{~m})$ is a test SAR image contains Wujiang river in China which is tested in [12]. The water area is extracted in Fig. 8(p) by their method. Our method gives satisfactory segmentation results in Fig. 8(n) with a more accurate and smoother boundary.

Fig. 9(a) shows a real MSTSAR SAR image of vehicle T72 which has been tested in [12]. This image mainly contains three regions: background, target and shadow. Fig. 9(b) shows the three-phase segmentation result of our proposed method. Fig. 9(c) and Fig. 9(d) display the membership functions $u_{1}$ and $u_{2}$. Our result is better than the result Fig. $9(\mathrm{~d})$ by method in [29] and competitive with the result Fig. 9(e) by method in [12].

\section{Conclusion}

A general multiphase fuzzy region competition model for texture image segmentation is proposed in this paper. There are two novelties. One is that a fuzzy membership function is introduced to represent a region for handling multiphase segmentation. The other is the use of kernel density estimation with optimal bandwidth to estimate the probability density function in each region represented by the fuzzy membership function. The proposed method is faster and easier to implement than the other curve evolution based methods. According to our experimental results, we find that our method is competitive with other state-of-the-art segmentation methods for texture images and SAR images.

\section{References}

[1] A. W. Bowman and A. Azzalini, Applied Smoothing Techniques for Data Analysis, Oxford University Press, 1997.

[2] X. Bresson, S. Esedoglu, P. Vandergheynst, J.-P. Thiran, S. Osher, Fast Global Minimization of the Active Contour/Snake Model, J. Math. Imaging and Vision. 28(2007), 151-167. 
[3] X. Bresson, T.F. Chan, Non-local unsupervised variational image segmentation models, UCLA cam report cam 08-67.

[4] K. Ni, X. Bresson, T.F. Chan and S. Esedoglu, Local Histogram Based Segmentation Using the Wasserstein Distance, UCLA cam report cam $08-47$.

[5] V. Caselles, R. Kimmel, and G. Sapiro, Geodesic active contours, Int. J. Computer Vision, 1(1997) 61-79.

[6] A. Chambolle, An algorithm for total variation minimization and applications, J. Math. Imaging and Vision. 20(2004) 89-97.

[7] T. F. Chan and L. A. Vese, Active contour without edges, IEEE Trans. Image Process. 10(2001) 266-277.

[8] T. F. Chan and L. A. Vese, Active contour and segmentation models using geometric PDE's for medical imaging, Malladi, R. (Ed.), Geometric Methods in Bio-Medical Image Processing, Series: Mathematics and Visualization, Springer, 2002.

[9] T. Chan, B. Sandberg, and L. Vese. Active contours without edges for vector-valued images. J. Visual Communication Image Representation. 11(2000) 130-141.

[10] T. F. Chan, S. Esedoglu, and M. Nikolova, Algorithms for finding global minimizers of image segmentation and denoising models, SIAM J. Appl. Math. 66(2006) 1632-1648.

[11] L. C. Evans and R. F. Gariepy, Measure theory and fine properties of functions, CRC Press, Boca Raton, FL, 1992.

[12] G. Gao, L. Zhao, J. Zhang, D. Zhou and J. Huang, A segmentation algorithm for SAR images based on the anisotropic heat diffusion equation, Pattern Recognition, 41(2008) 3035-3043.

[13] N. Houhou, J.P.Thiran and X. Bresson, Fast texture segmentation model based on the shape operator and active contour, IEEE Conference on Computer Vision and Pattern Recognition, CVPR 2008. 
[14] Y. Huang, M. Ng and Y. Wen, A Fast Total Variation Minimization Method for Image Restoration, SIAM J. Multiscale Modeling and Simulation. 7(2008) 774-795.

[15] G. T. Herman and B. M. Carvalho, Simultaneous Fuzzy Segmentation of Medical Images Handbook of Biomedical Image Analysis chapter 12, Springer US, 2007.

[16] J. Quan, X. Wen, X. Xu, Multiscale probabilistic neural network method for SAR image segmentation, Appl. Mathematics and Computation. 205(2008) 578-583.

[17] J. Kim, J. Fisher, A. Yezzi, M. Cetin, and A. Willsky, A Nonparametric Statistical Method for Image Segmentation Using Information Theory and Curve Evolution. IEEE Trans. Image Process. 14(2005) 1486-1502.

[18] Mila Nikolova, Selim Esedoglu, and Tony F. Chan, Algorithms for Finding Global Minimizers of Image Segmentation and Denoising Models, SIAM J. Appl. Math. 66(2006) 1632-1648.

[19] B. Mory and R. Ardon, Fuzzy region competition: a convex two-phase segmentation framework, F. Sgallari, A. Murli, and N. Paragios (Eds.) SSVM 2007, LNCS 4485, pp. 214-226, 2007.

[20] B. Mory and R. Ardon, Variational segmentation using fuzzy region competition and local non-parametric probability desity functions, ICCV 2007, IEEE $11^{\text {th }}$ international conference on computer vision, pp. $1-8$.

[21] D. Mumford and J. Shah, Optimal approximations by piecewise smooth functions and associated variational problems, Comm. on Pure and Applied Mathematics. 42(1989) 577-685.

[22] S. Osher and N. Paragios, Geometric Level Set Methods in Imaging Vision and Graphics, Springer Verlag, 2003.

[23] E. Parzen: On the estimation of a probability density function and mode, Annals of Mathematical Statistics. 33(1962) 1065-1076.

[24] N. Paragios, and R. Deriche: Geodesic active regions: A new paradigm to deal with frame partition problems in computer vision, J. Visual Communication and Image Representation. 13(2002) 249-268. 
[25] M. Rousson, T. Brox and R. Deriche, Active unsupervised texture segmentation on a diffusion based feature space, In Proc. 2003 IEEE Computer Society Conference on Computer Vision and Pattern Recognition, CVPR03, June 2003.

[26] L.I. Rudin, S. Osher and E. Fatemi, Nonlinear total variation based noise removal algorithms. Physica D. 60(1992) 259-268.

[27] C. Sagiv, N. A. Sochen, and Y. Y. Zeevi. Texture segmentation via a diffusion-segmentation scheme in the gabor feature space. In Proc. Texture 2002, 2nd International Workshop on Texture Analysis and Synthesis, Copenhagen, June 2002.

[28] J. Shen, A stochastic-variational model for soft Mumford-Shah segmentation, Int. J. Biomedical Imaging, 2006, Article ID 92329, pp. 1-14.

[29] R.A. Weisenseel, W.C. Karl, D.A. Castanon, R.C. Brower, MRF-based algorithms for segmentation of SAR images, IEEE Proceeding of the 1998 International Conference on Image Processing, 3(1998), pp. 770774.

[30] A. Yezzi, Jr., A. Tsai, and A. Willsky, A statistical approach to snakes for bimodal and trimodal imagery, in Int. Conf. Computer Vision. 1999, pp. 898-903.

[31] S. C. Zhu and A. Yuille, Region competition: Unifying snakes, region growing, and bayes/mdl for multiband image segmentation, IEEE Trans. Pattern Anal. Machine Intell. 18(1996) 884-900. 


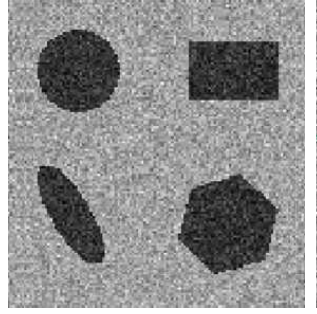

(a)

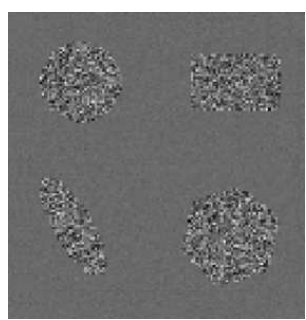

(d)

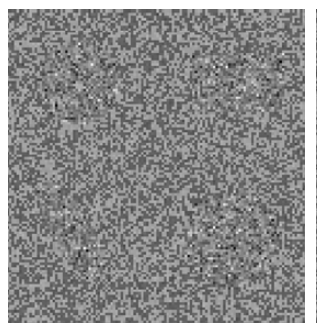

(g)

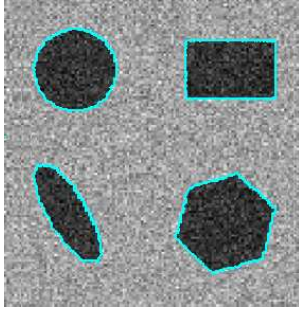

(b)

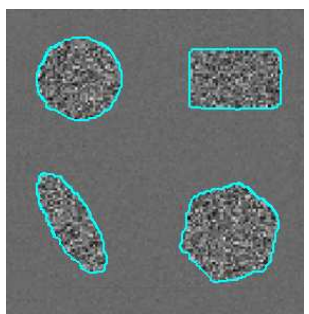

(e)

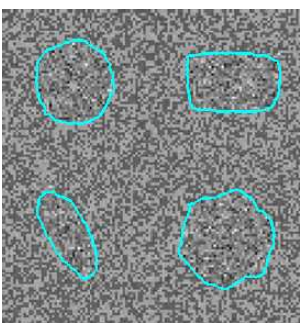

(h)

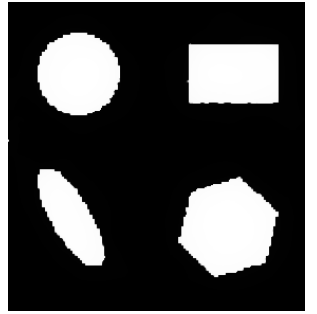

(c)

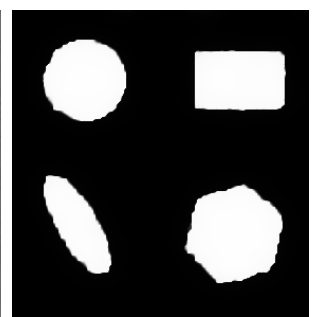

(f)

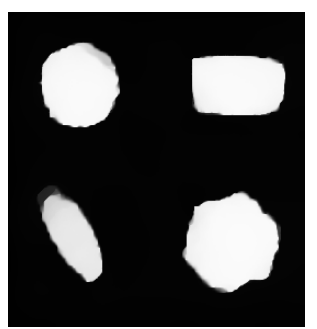

(i)

Figure 2: Two-phase image segmentation. The First column: synthetic images; the Second column: segmentation results; the Third column: membership functions $u_{1}$. 


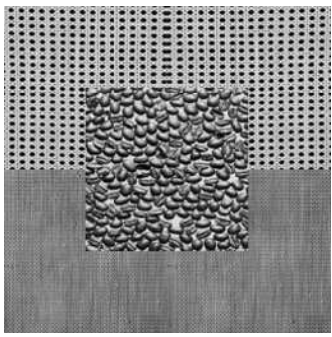

(a)

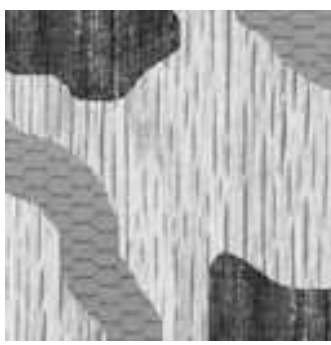

(e)

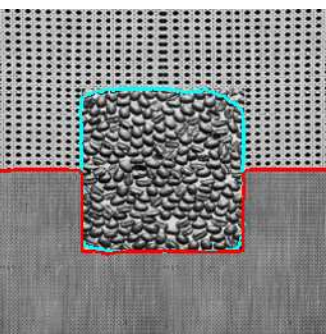

(b)

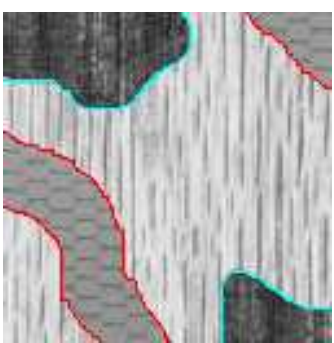

(f)

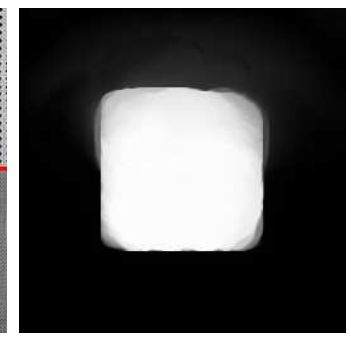

(c)

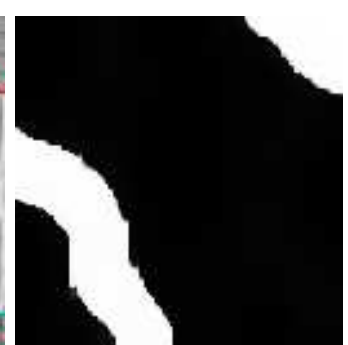

(g)

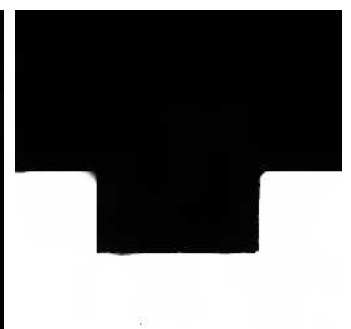

(d)

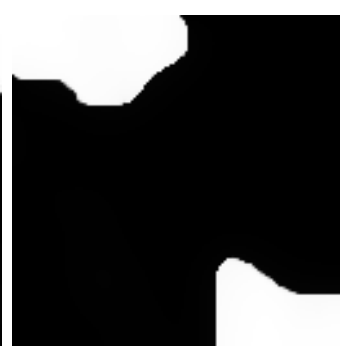

(h)

Figure 3: Three-phase image segmentation. The First column: synthetic images; the Second column: segmentation results; the Third column: membership functions $u_{1}$; the Fourth column: membership functions $u_{2}$. 


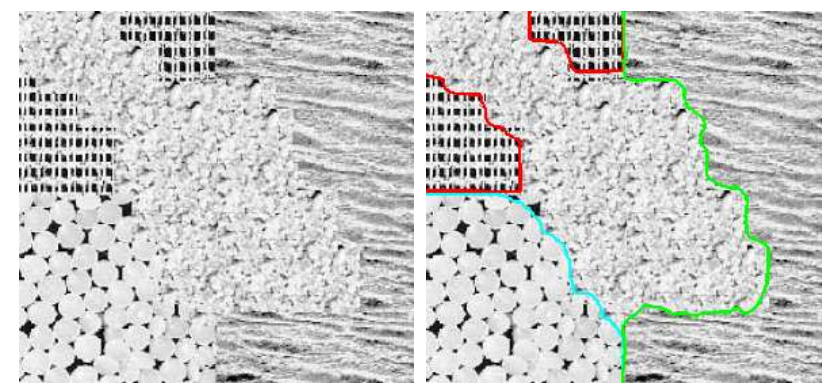

(a)

(b)

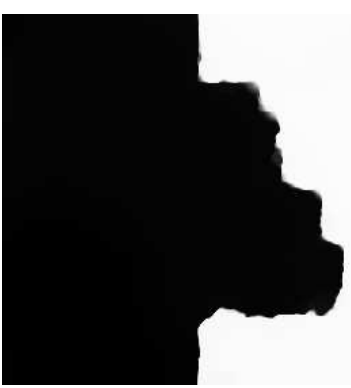

(c)

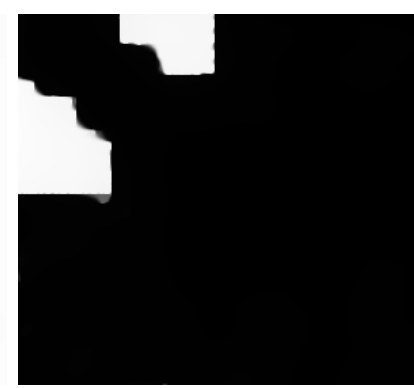

(d)

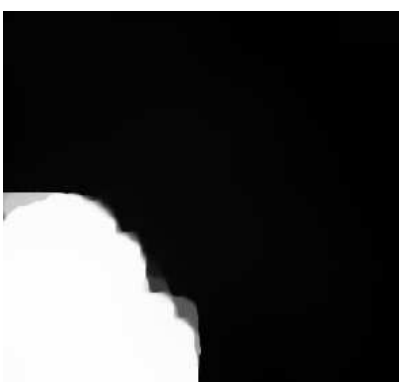

(e)

Figure 4: Four-phase image segmentation. (a) synthetic image; (b) segmentation result; (c) membership function $u_{1}$; (d) membership function $u_{2}$; (d) membership function $u_{3}$. 


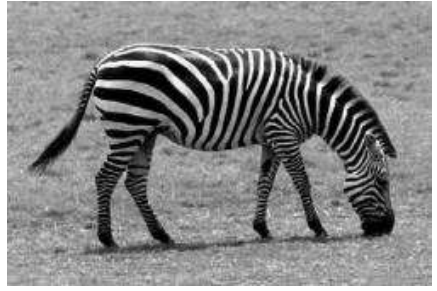

(a)

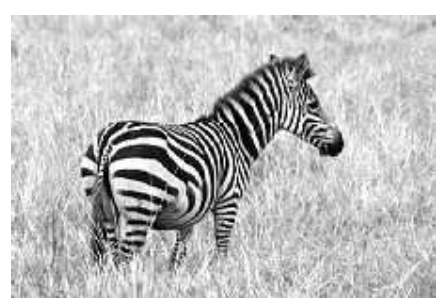

(d)

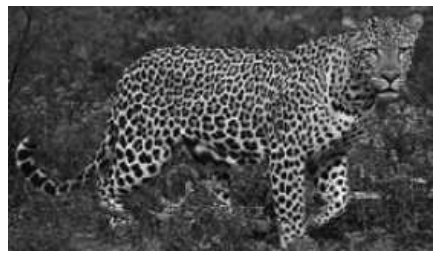

(g)

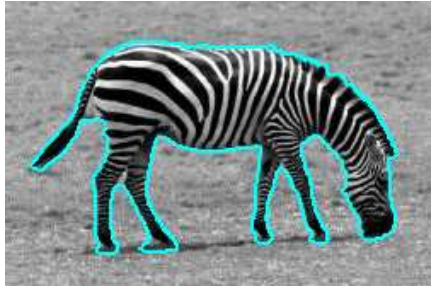

(b)

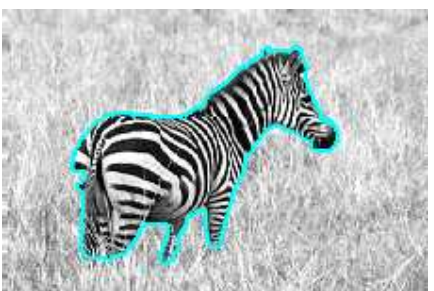

(e)

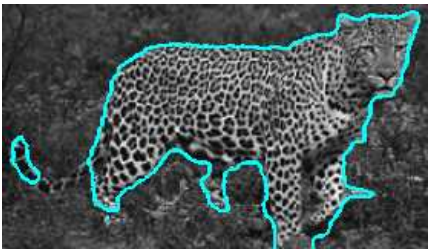

(h)

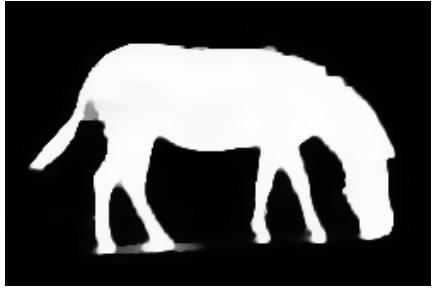

(c)

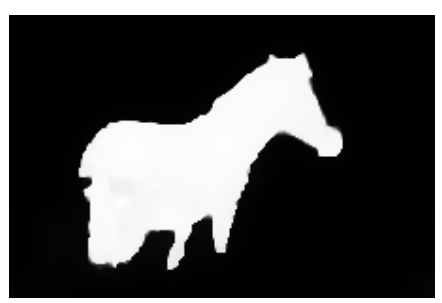

(f)

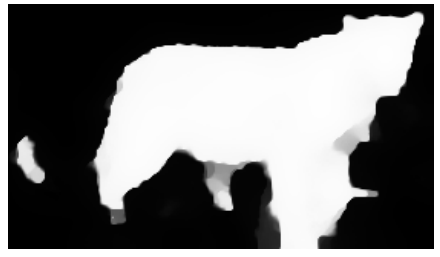

(i)

Figure 5: Two-phase image segmentation. The First column: natural images; the Second column: segmentation results; the Third column: membership functions $u_{1}$

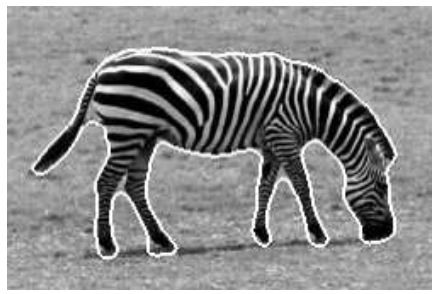

(a)

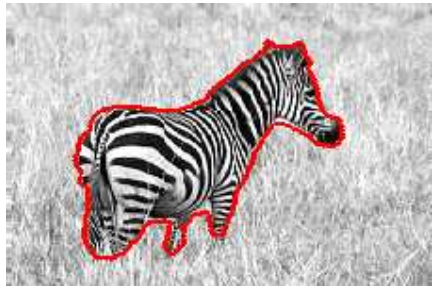

(b)

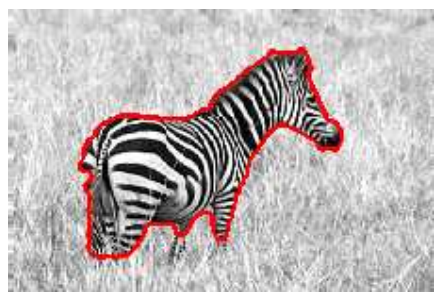

(c)

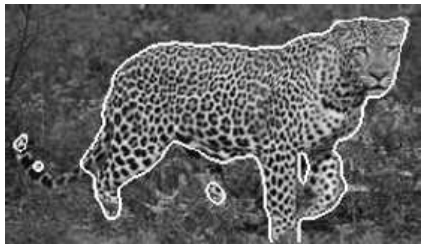

(d)

Figure 6: The Comparison. (a), (b) and (d) by the method in [25]; (c) by the method in [20] with energy (3). 


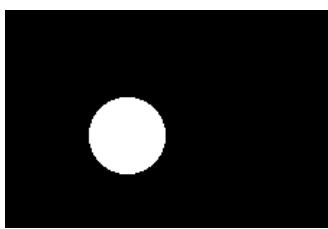

(a) initial

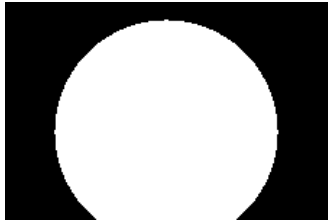

(e) initial

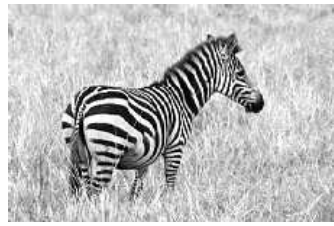

(i) initial

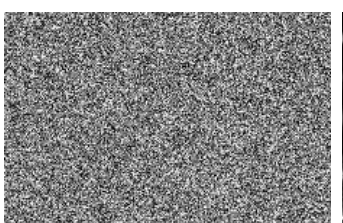

(m) initial

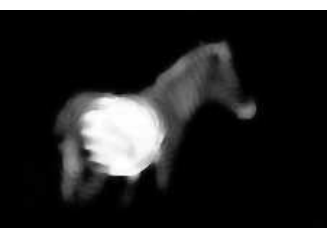

(b) intermediate

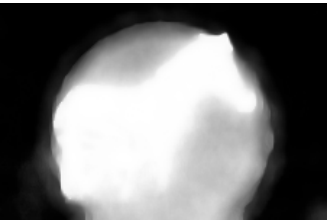

(f) intermediate

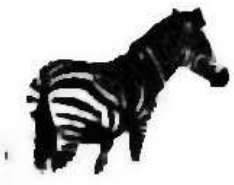

(j) intermediate

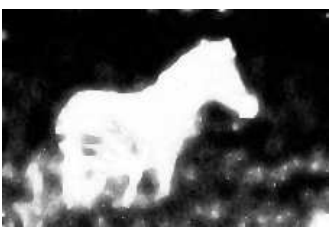

(n) intermediate

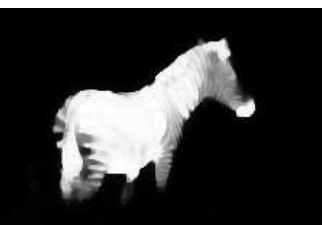

(c) intermediate

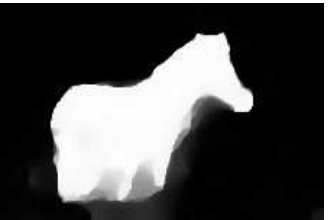

(g) intermediate

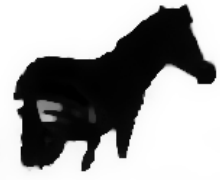

(k) intermediate

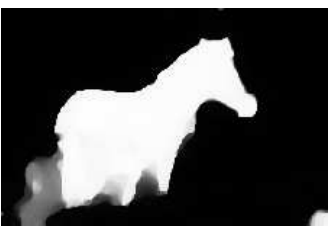

(o) intermediate

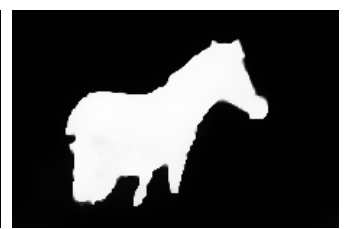

(d) final

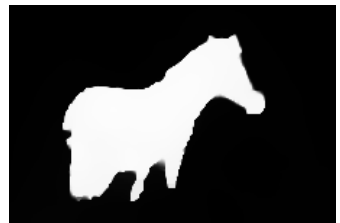

(h) final

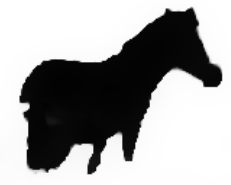

(1) final

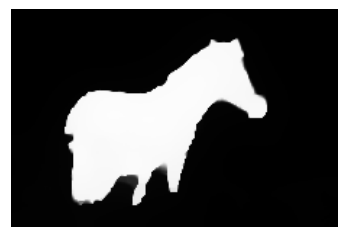

(p) final

Figure 7: Evolution of the membership functions with different initializations on a zebra image. The final results from top to bottom are obtained at iterations 105, 270, 150 and 240 respectively. 


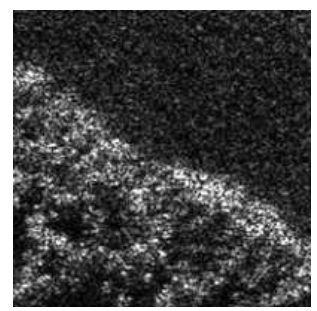

(a)

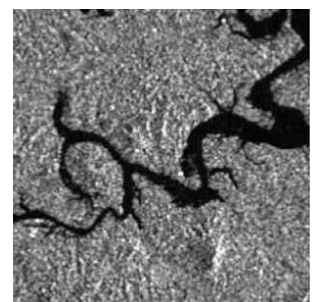

(e)

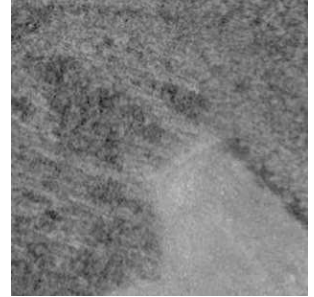

(i)

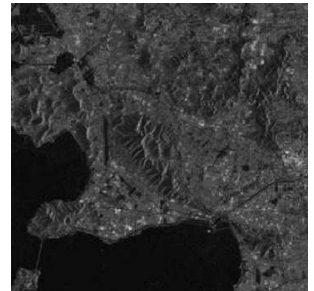

(m)

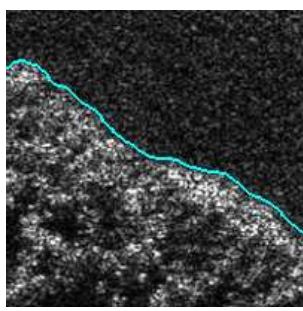

(b)

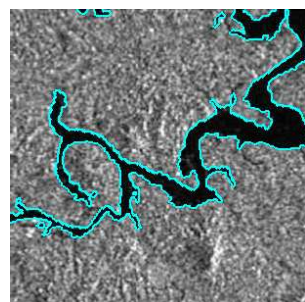

(f)

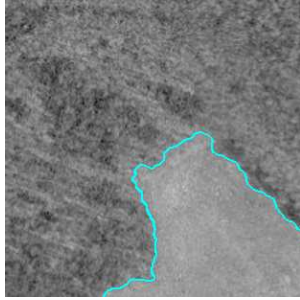

(j)

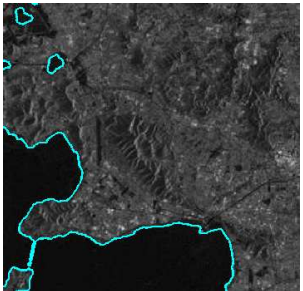

(n)

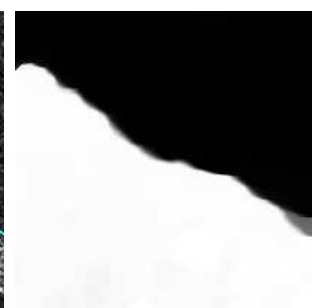

(c)

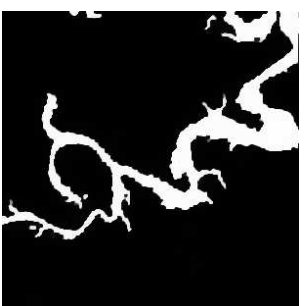

(g)

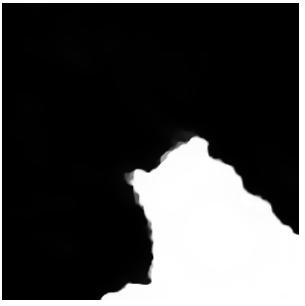

(k)

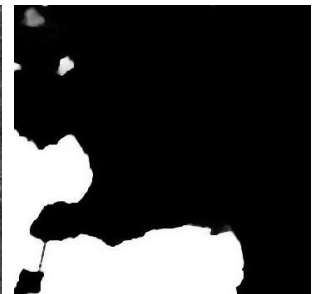

(o)

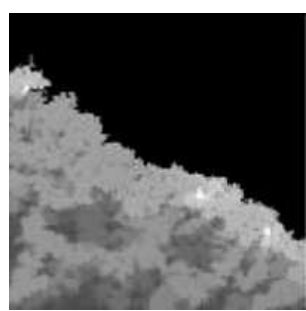

(d)

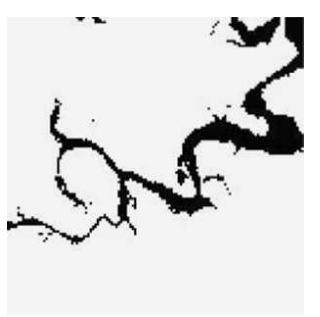

(h)

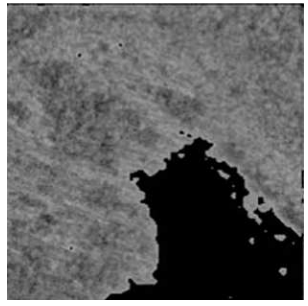

(1)

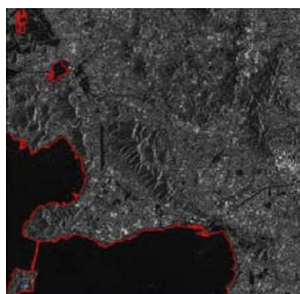

(p)

Figure 8: Two-phase SAR image segmentation. The First column: SAR images; the Second column: segmentation results; the Third column: membership functions $u_{1}$; the Fourth column: segmentation results of the other methods: (d) by the method in [15]; (h) and (l) by the method in [16]; (p) by the method in [12]. 


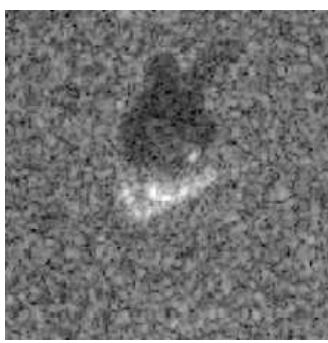

(a)

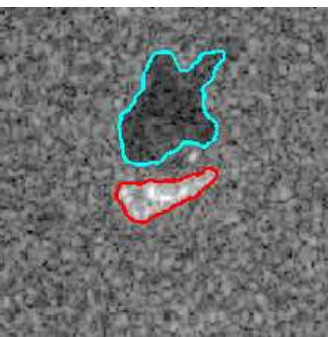

(b)

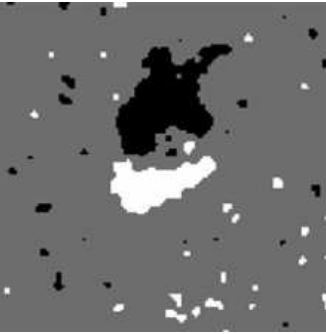

(e)

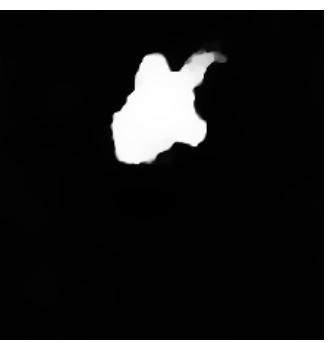

(c)

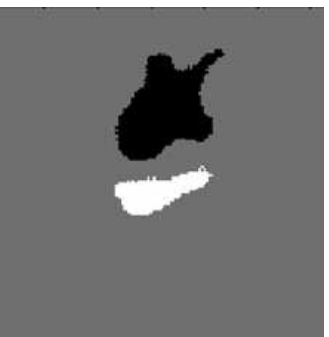

(f)

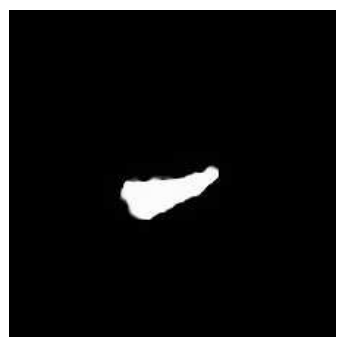

(d)

Figure 9: Three-phase SAR image segmentation. (a) SAR image; (b) segmentation result by the proposed method; (c) membership function $u_{1}$; (d) membership function $u_{2}$; (e) segmentation result by method in [29]; (f) segmentation result by method in [12]. 